Original Research Paper

\title{
Anatomical Changes of Akar Putra Chicken Digestive System after Partial Ablation of Uropygial Gland
}

\author{
${ }^{3,1}$ Hasan Saad Jawad, ${ }^{1}$ Lokman Hakim bin Idris, ${ }^{1}$ Md Zuki bin Bakar and ${ }^{2}$ Azhar bin Kassim \\ ${ }^{I}$ Department of Veterinary Preclinical Sciences, Faculty of Veterinary Medicine, \\ Universiti Putra Malaysia, Malaysia \\ ${ }^{2}$ Department of Animal Sciences, Faculty of Agriculture, Universiti Putra Malaysia, Malaysia \\ ${ }^{3}$ Department of Veterinary Anatomy, Histology and Embryology, Faculty of Veterinary Medicine, \\ University of Baghdad, Iraq
}

Article history

Received: 06-06-2015

Revised: 16-07-2015

Accepted: 17-10-2015

Corresponding Author:

Hasan, S.A. Jawad

Department of Veterinary

Preclinical Sciences, Faculty of

Veterinary Medicine,

Universiti Putra Malaysia,

Malaysia

Email: dr.hassan198366@yahoo.com

\begin{abstract}
The current study proposes anatomical changes in the digestive system of 120 Akar Putra chicken after surgical removing of auropygial gland. The experiment comprised of five treatments ( 24 chicken/treatment), with 3 replicates ( 8 chicken/replicate). The experimental treatments consist of a control treatment $\mathrm{T} 1$; partial ablation of the uropygial gland were applied on T2, T3, T4 and T5 treatments at 3, 4, 5 and 6 weeks of age respectively. The results presents that Partial Uropygialectomy (PU) treatments in males had $(\mathrm{p}<0.01)$ longer esophagus 9.9-16.2\%, proventriculus $11.1-34.4 \%$, gizzard $26.7-220 \%$, pancreas $0-20.4 \%$, jejunum 4.9-26.1 and colon 18.1-60.6 than the control group counterparts. Furthermore, females of PU treatments had $(\mathrm{p}<0.01)$ longer esophagus 6.8$22.3 \%$, pancreas $8.3-33.3 \%$ and cecum $13-26 \%$ compared with females in control. In contrast, total GIT weight were 21.2 to $78.8 \%$ heavier $(\mathrm{p}<0.01)$ in males of PU treatments, mainly in the esophagus, duodenum, pancreas, jejunum, ilium and cecum. No significant impact $(p>0.05)$ between treatments was recorded in the total females' Gastrointestinal Tract (GIT) weight; however, females of PU treatments had $5.9-41.2 \%$ heavier ilium $(p<0.01)$ and $11.1-77.8 \%$ heavier cecum $(p<0.01)$. Total GIT density, show no significant different $(p>0.05)$ between treatments in females. Nevertheless, it was highly significant different $(p<0.01)$ for males of PU treatments, mainly in esophagus $(\mathrm{p}<0.05)$, gizzard $(\mathrm{p}<0.05)$, pancreas $(p<0.01)$ and cecum $(p<0.05)$. In conclusion; the results of current study investigated that partial ablation of the uropygial gland had positive effects on the anatomical observations of most digestive system parts.
\end{abstract}

Keywords: Avian Gland, Uropygial Gland, Akar Putra Chicken

\section{Introduction}

Akar Putra is a local Malaysian chicken, created in the University of Putra Malaysia by Assoc. Prof. A.B. Kassim. It has arobust growing process than their parents because the maturation period is shorter (less than 13 weeks). It can lay 120-200 eggs per year and it has more resistance to diseases (Jawad et al., 2015).

The digestive tract of chickens conveys food to the stomach: This system comprises, the crop, an expansion of the esophagus, located in the lower neck area, the glandular stomach (proventriculus), the muscular stomach (gizzard) and intestines. The length and weight of the small intestine varied between the different species of birds (Hassouna, 2001). Differential development of the absorptive epithelium may be responsible for changes in absorption capacity of birds (de Verdal et al., 2010).

Uropygial gland is the only subcutaneous gland in bird's body (Mclelland, 1993). It is located on the base of the tail, dorsally between the fourth caudal vertebrae and the pygostyle (Lucas and Stettenheim, 1972; Sawad, 2006). The function of the uropygial gland is still a subject of controversy. There are many accepted functions of gland secretions like conferring waterrepellent properties on the feather coat and maintaining 
the suppleness of it. Furthermore, it's proposed to be associated to pheromone production, control of plumage hygiene, thermal insulation and defense against predators (Jacob, 1992; Montalti et al., 2006; 1998; Soler et al., 2012; Vincze et al., 2013). Uropygial gland is completely absent in Struthionidae, Rheidae, Casuaridae, Dromaidae and in a few species of Columbidae and Psittacidae (Johnston, 1988). Montalti and Salibián (2000) mentioned that the oil of uropygial gland is not important to the birds who do not have it and uropygial gland in some of the birds is non active Goodwin (1983). Moyer et al. (2003) explained that the birds which do not have uropygial gland use dusters bath to keep and clean their feather.

Many studies confirmed that meat chicken breeds nowadays characterized by super-fast growth and a high efficiency of the feed conversion ratio because of intense genetic election. Wepruk and Church (2003) observed that the mean body weight of a broiler at the age of 63 days in 1976 was two $\mathrm{kg}$, while the same average body weight was arrived at by the age of 35 days in 2001 . These improvements in the growth rate will be reflected negatively on the disease resistance and immune response of these birds, because a negative genetic link coefficient was observed between the growth speeds and immune response (Qureshi and Havenstein, 1994). In this context, mortality ratio increased in these strains of birds has happened due to increase their susceptibility to bacterial diseases and metabolic diseases. These occurred as a consequence of irregular metabolous processes, an imbalance in the acid-base balancebaseband Acid-base balance of body fluids, such as ascites disease, Sudden-Death Syndrome (SDS) and increased skeletal disorders like leg abnormalities. It has been scientifically proven that highest rates of those pathological conditions were shown in flockand individual rapid growth chickens at 3 and 4 weeks of age (Robinson et al., 1992; Leeson and Summer, 1997; Julian, 1997; 1998; Gonzales et al., 1998). Based on the limitation of the problem, the researcher innovating a safe technique to raise the level of digestive system anatomy in poultry generally and local Malaysian chicken (Akar Putra) particularly without using genetic improvement methods. Furthermore, this research another two significances: (1) To identify the information regarding the anatomy of Akar Putra chicken digestive system because it's new specious of chicken. (2) To identify the scientific information about PU treatment operation effect on the digestive system anatomy of the chicken.

\section{Materials and Methods}

\section{Research Design}

The research design conducted with Complete Random Sampling Design (RAL) with 5 variables, divided into two groups: 1 control group and 4 treatment variables with different ages of applying Partial Uropygialectomy. The research were conducted in University of Putra Malaysia (UPM) from $15^{\text {th }}$ December 2014 to 15 th March 2015. This study was approved by Institutional Animal Care and Use Committee (IACUC) certificate number R070.

Research variables: The variables which were observed during this research were.

Independent variables: The variation of Partial Uropygialectomy (PU) in following requirements:

T1: Group without PU operation (control group)

T2: Group which was PU applied at week 3

T3: Group which was PU applied at week 4

T4: Group which was PU applied at week 5

T5: Group which was PU applied at week 6

\section{Dependent Variable}

The esophagus, proventriculus, gizzard, duodenum, pancreas, jejunum, ilium, cecum, colon and rectum were excised, cleaned and separately weighed. In addition, their lengths were measured individually. The weight: Length ratios of each part was calculated as an indicator of its density. In the same regard, the relative weight and length of each part of GIT with the total weight and length of GIT were compared between treatments. Moreover, the relative weights of these parts with the total live body weight were calculated.

\section{Population and Sample of the Research}

- Research populations were a Local Malaysian chicken (Akar Putra)

- Research samples were 120 Akar Putra chicken randomly assigned to five treatment groups by 24 (12 male and 12 female) chickens per treatment and each treatment consist of three replicates of 8 chickens (4 males and 4 females)

\section{Tools and Materials}

Lidocaine, 70\% Alcohol, Iodine, scalpels and blades were used to apply during PU operation. An electronic balance (precision $=1 \mathrm{~g}$ ) was used to measure the live body weight and GIT parts weight of the experimental birds. The lengths of GIT parts were measured with a tape measure $( \pm 1 \mathrm{~mm})$.

The materials used in this research consisted of a Day-Old Chick of Local Malaysian chicken (Akar Putra) Strain. The birds were given ad libitum access to feed and water. The birds were rearedin cages with 8 birds (4 males and 4 females) per pen ( $5 " \times 4 " \times 1.5 ")$. Feed was offered ad libitum (1-13 days: Starter; 14 day-slaughter: finisher) and water was freely available at all times during the trial period. Furthermore, constant lighting 
and continuous ventilation were provided and all the birds were kept under uniform management conditions throughout the experimental period of 12 weeks.

\section{Procedure of the Research}

This research was conducted in several stages as follows:

- Partial Uropygialectomy operation was applied as the following steps: (A) Bird's restraint. (B) Local anesthesia using lidocaine HCL (4 mg kg-1) SQ. (C) Removing the uropygial gland partially (half lobes, half isthmus and papillae) by scalpel which sterilized by $70 \%$ alcohol before use. (D) After removing the gland, the incision area was sterilized with Iodine

- On the last day of experiment, 12 birds (closest to the mean treatments' body weight) were selected from each treatment group (2 males and 2 females/replicate) and withdrawn from feed overnight to facilitate gut clearance. After obtaining the live body weight, the birds were euthanized by an intravenous (cutaneous ulnar vein) injection of sodium pentobarbitone $(80 \mathrm{mg}$ $\mathrm{kg}^{-1}$ ) (Mitchell and Smith, 1991). The digestive tract segments from the esophagus to the rectum was carefully excised, identified and analyzed based on Nasrin et al. (2012) as following:

- Esophagus extends from the glottis at the posterior end of the pharynx, through the neck and thorax to join with the glandular stomach

- Proventriculus located caudal to the crop

- Gizzard placed partly between the lobes and to some extent behind the left lobe of the liver

- Duodenum extends from pylorus to the end of the pancreatic loop

- Pancreas located in the duodenal fold

- Jejunum extends from the pancreatic loop to Meckel's diverticulum

- Ilium extends from Meckel's diverticulum to the ileo-caeca junction

- Cecum: The two caeca were blind pouches and extend along the line of the small intestine towards the liver having proximal and distal part and were closely attached to the small intestine along their length by the mesentery

- Colon and rectum: They are passing between the ileo-cecal junction and the cloaca

Digestive segments were cleaned, separately weighed and their lengths were measured individually. The weight: Length ratio of each partwas calculated as an indicator of its density (Taylor and Jones, 2004). In the same regard, the relative weight and length, of each part of GIT with the total weight and length of GIT, were compared with the treatments. Further more, the relative weights of these parts with the total live body weight were calculated.

Variation ratio of the GIT morphometrical characteristics had recorded based on the formula which reported by (Jawad et al., 2015):

$$
((\mathrm{A}-\mathrm{B}) / \mathrm{B})^{*} 100
$$
A: Treatment data
B: Control group data

\section{Data Analysis}

Data generated from experiment was carried out in a complete randomized design (Steel and Torrie, 1980). These data were subjected to ANOVA using Genstat (2003). If the treatment significantly affected the chicken, LSD and Duncan (1955) Multiple Range would be applied (DRMT) (Gaspers, 1991). The significant differences among means were determined by using Duncan (1955) multiple range tests. Differences among treatment means were compared at $\mathrm{p}<0.01$ and $\mathrm{p}<0.05$ levels.

\section{Results}

\section{GIT Parts' Length}

Table 1 shows the linear measurements' descriptive statistics of males' GIT parts in PU treatments and control group. Males in T4, T3, T2 and T5 treatments showshighly significant $(\mathrm{p}<0.01)$ effect compared with the control group in esophagus length. T4 treatment recorded higher $(\mathrm{p}<0.01)$ esophagus length $21.5 \mathrm{~cm}$. The males' proventriculus of PU treatments was longer compared with the control group, especially in T2 treatment, which recorded $4.033 \mathrm{~cm}$. However, no significant impact was indicated between T2, T5, T3 and T4. High significantly affect $(p<0.01)$ in gizzard length capacity was reported in $\mathrm{T} 5(4.8 \mathrm{~cm})$. The pancreas was longer in T3 $(10,833 \mathrm{~cm}), \mathrm{T} 4(10.333 \mathrm{~cm})$ and T2 $(10.267 \mathrm{~cm})$ compared with T5 $(9 \mathrm{~cm})$ and the control $(9$ $\mathrm{cm})$. Whereas, all PU treatments recorded higher values in jejunum length and colon length also in the total GIT length compare with the control group. Although there is no perceptible difference $(p>0.05)$ between T5, T2 and $\mathrm{T} 3$ treatments in jejunum length and between T2, T4 and $\mathrm{T} 3$ in colon length, moreover, between T5, T2 and T2, T4 in complete GIT length.

Relative length of males GIT parts than the total GIT length was presented in Table 2. Only the esophagus, gizzard and pancreas revealed $(\mathrm{p}<0.01)$ significant different between the treatments. The superiority order in the relative esophagus length trait was T4, T3, T1, T2 and T5 and the values were as following: 10.464, 10.321, 10.028, 9.596 and $9.215 \mathrm{~cm}$ correspondingly. While, in relative gizzard's length trait was T5, T2, T3, T4 and T1 and the values were: $2.183,1.061,1.052,0.925$ and $0.813 \mathrm{~cm}$ respectively. 
Lowest value in relative pancreatic length was reported in T5 $(4.079 \mathrm{~cm})$ and the highest value was in T3 $(5.325 \mathrm{~cm})$ followed by T4 $(5.032 \mathrm{~cm}), \mathrm{T} 1(4.879 \mathrm{~cm})$ and T2 $(4.744$ $\mathrm{cm})$. However, no significant different between T3, T4, T1 and $\mathrm{T} 2$ was indicated.

Table 3 shows the length variation in females' digestive tract segments between the treatments. The effect of the surgical removing of the uropygial gland at week 6 of age was prominent through relevant $(p<0.01)$ superiority in esophagus length over the rest of the transactions. Not as much value for the pancreas length trait was recorded in $\mathrm{T} 4$ and $\mathrm{T} 5$ treatments despite the absence of a significant difference between them and the value of the pancreas length in the control group. While the cecum length was prominent in $\mathrm{T} 2$, T5 and T4 treatments and less pronounced in T3 treatment compared with the control treatment. Regarding on the females' relative length of GIT parts than the total GIT length was shown in Table 4. Only pancreas revealed significantly different in T2 (5.442) and T3 (5.103) then followed by T1 (4.913), T4 (4.672) and T5 (4.398) respectively.

\section{GIT Parts' Weight}

Six segments weights of the digestive system in both of males and females were reported high significant $(p<0.01)$ different between the treatments. They were esophagus, duodenum, pancreas, jejunum, ilium and cecum weights in males. While, they were esophagus, duodenum, ilium and cecum weights in females.

Table 5 provides a comprehensive look at the averages and standard error values of GIT parts weights for males in all the treatments. Males of PU treatments have outperformed than the control group in $(p<0.01)$ significant vary digestive system segments' weights such as esophagus, pancreas, jejunum, ilium and cecum. Second treatment recorded high significantly $(p<0.01)$ different in the average's weights of males $1673 \mathrm{gm}$ and followed by T5, T3, T4 and T1. Their averages' weights were 1639.333, 1598.333, 1577.667 and $1390.667 \mathrm{gm}$ correspondingly. Esophagus weight in T2 was heavier $(p<0.01)$ than the rest of the treatments; however, no significant different between T2, T5 and T3 also between T4 and T1 was observed.

Table 1. Mean $( \pm$ S.E.) males GIT parts length $(\mathrm{cm})$ of partial uropygialectomy treatments.

Treatments

\begin{tabular}{llllll} 
GIT parts & T1 (control) & T2 & T3 & T4 & T5 \\
\hline Eso. & $18.5 \pm 0.764^{\mathrm{b}}$ & $20.767 \pm 0.145^{\mathrm{a}}$ & $21 \pm 0.577^{\mathrm{a}}$ & $21.5 \pm 0.289^{\mathrm{a}}$ & $20.333 \pm 0.333^{\mathrm{a}}$ \\
Pro. V. & $3 \pm 0.289^{\mathrm{b}}$ & $4.033 \pm 0.145^{\mathrm{a}}$ & $3.833 \pm 0.167^{\mathrm{a}}$ & $3.333 \pm 0.167^{\mathrm{ab}}$ & $3.933 \pm 0.296^{\mathrm{a}}$ \\
Giz. & $1.5 \pm 0^{\mathrm{b}}$ & $2.3 \pm 0.153^{\mathrm{b}}$ & $2.133 \pm 0.318^{\mathrm{b}}$ & $1.9 \pm 0.1^{\mathrm{b}}$ & $4.8 \pm 0.458^{\mathrm{a}}$ \\
Deu. & $22 \pm 1.155$ & $26.2 \pm 1.8$ & $25 \pm 0.577$ & $24.833 \pm 1.364$ & $23.333 \pm 0.333$ \\
pancreas & $9 \pm 0^{\mathrm{b}}$ & $10.267 \pm 0.267^{\mathrm{a}}$ & $10.833 \pm 0.726^{\mathrm{a}}$ & $10.333 \pm 0.167^{\mathrm{a}}$ & $9 \pm 0^{\mathrm{b}}$ \\
Juj. & $43.833 \pm 1.641^{\mathrm{c}}$ & $52.367 \pm 2.709^{\mathrm{ab}}$ & $48.667 \pm 1.856^{\mathrm{ab}}$ & $46 \pm 1^{\mathrm{bc}}$ & $55.267 \pm 3.688^{\mathrm{a}}$ \\
Ilium & $48.66 \pm 2.404$ & $56.667 \pm 2.455$ & $50.833 \pm 4.285$ & $54 \pm 3.786$ & $60.567 \pm 1.598$ \\
Cecum & $29 \pm 0.577$ & $33 \pm 0.577$ & $30.9 \pm 2.951$ & $33 \pm 1$ & $31.067 \pm 0.581$ \\
Colon & $5.167 \pm 0.333^{\mathrm{c}}$ & $6.667 \pm 0.333^{\mathrm{b}}$ & $6.1 \pm 0.208^{\mathrm{bc}}$ & $6.133 \pm 0.696^{\mathrm{bc}}$ & $8.3 \pm 0.351^{\mathrm{a}}$ \\
Rectum & $3.833 \pm 0.167$ & $4.2 \pm 0.651$ & $4.3 \pm 0.7$ & $4.5 \pm 0.5$ & $4.167 \pm 0.167$ \\
Total & $184.5 \pm 2.021^{\mathrm{d}}$ & $220.767 \pm 3.775^{\mathrm{a}}$ & $205.533 \pm 4.218^{\mathrm{bc}}$ & $216.467 \pm 3.039^{\mathrm{ab}}$ & $203.6 \pm 4.212^{\mathrm{c}}$ \\
\hline
\end{tabular}

Mean values with common superscript in row differ significantly $(\mathrm{p}<0.01)$

Table 2. Relative length (\%) of males GIT parts than total GIT length of partial uropygialectomy treatments.

\begin{tabular}{|c|c|c|c|c|c|}
\hline \multirow[b]{2}{*}{ GIT parts } & \multicolumn{4}{|c|}{ Treatments } & \multirow[b]{2}{*}{$\mathrm{T} 5$} \\
\hline & T1 (control) & $\mathrm{T} 2$ & T3 & $\mathrm{T} 4$ & \\
\hline Eso. & $10.028 \pm 0.412^{\mathrm{abc}}$ & $9.596 \pm 0.103^{\mathrm{c}}$ & $10.321 \pm 0.325^{\mathrm{ab}}$ & $10.464 \pm 0.074^{\mathrm{a}}$ & $9.215 \pm 0.197^{c}$ \\
\hline Pro. V. & $1.626 \pm 0.157$ & $1.865 \pm 0.079$ & $1.886 \pm 0.103$ & $1.623 \pm 0.088$ & $1.786 \pm 0.162$ \\
\hline Giz. & $0.813 \pm 0.009^{\mathrm{b}}$ & $1.061 \pm 0.057^{\mathrm{b}}$ & $1.052 \pm 0.167^{\mathrm{b}}$ & $0.925 \pm 0.052^{\mathrm{b}}$ & $2.183 \pm 0.247^{\mathrm{a}}$ \\
\hline Deu. & $11.914 \pm 0.506$ & $12.117 \pm 0.515$ & $12.292 \pm 0.409$ & $12.099 \pm 0.752$ & $10.58 \pm 0.331$ \\
\hline Pancreas & $4.879 \pm 0.053^{\mathrm{a}}$ & $4.744 \pm 0.134^{\mathrm{a}}$ & $5.325 \pm 0.364^{\mathrm{a}}$ & $5.032 \pm 0.131^{\mathrm{a}}$ & $4.079 \pm 0.07^{\mathrm{b}}$ \\
\hline Juj. & $23.775 \pm 1.061$ & $24.167 \pm 0.946$ & $23.887 \pm 0.478$ & $22.382 \pm 0.23$ & $24.992 \pm 1.272$ \\
\hline Ilium & $26.367 \pm 1.162$ & $26.166 \pm 0.896$ & $24.914 \pm 1.68$ & $26.221 \pm 1.326$ & $27.427 \pm 0.298$ \\
\hline Cecum & $15.721 \pm 0.336$ & $15.254 \pm 0.404$ & $15.227 \pm 1.649$ & $16.069 \pm 0.588$ & $14.086 \pm 0.45$ \\
\hline Colon & $2.799 \pm 0.165$ & $3.081 \pm 0.164$ & $2.995 \pm 0.06$ & $2.994 \pm 0.384$ & $3.761 \pm 0.164$ \\
\hline Rectum & $2.077 \pm 0.085$ & $1.949 \pm 0.329$ & $2.1 \pm 0.307$ & $2.191 \pm 0.248$ & $1.891 \pm 0.108$ \\
\hline
\end{tabular}

Mean values with common superscript in row differ significantly $(\mathrm{p}<0.01)$ 
$\underline{\text { Table 3. Mean }( \pm \text { S.E.) females GIT parts length }(\mathrm{cm}) \text { of partial uropygialectomy treatments. }}$ Treatments

\begin{tabular}{|c|c|c|c|c|c|}
\hline GIT parts & T1 (Control) & $\mathrm{T} 2$ & $\mathrm{~T} 3$ & $\mathrm{~T} 4$ & T5 \\
\hline Eso. & $15.667 \pm 0.333^{c}$ & $16.733 \pm 0.176^{\mathrm{bc}}$ & $18 \pm 0.577^{\mathrm{ab}}$ & $18.333 \pm 0.882^{a b}$ & $19.167 \pm 1.093^{\mathrm{a}}$ \\
\hline Pro. V. & $3.667 \pm 0.333$ & $3.733 \pm 0.145$ & $3.2 \pm 0.115$ & $3.333 \pm 0.167$ & $3.433 \pm 0.186$ \\
\hline Giz. & $1.5 \pm 0$ & $1.867 \pm 0.067$ & $2.833 \pm 0.601$ & $1.5 \pm 0$ & $3.167 \pm 0.726$ \\
\hline Deu. & $20.333 \pm 1.333$ & $25.067 \pm 1.933$ & $23.833 \pm 0.441$ & $23.833 \pm 1.364$ & $25.267 \pm 2.267$ \\
\hline pancreas & $8 \pm 0^{c}$ & $10.667 \pm 0.333^{a}$ & $9.567 \pm 0.296^{\mathrm{b}}$ & $8.833 \pm 0.441^{b c}$ & $8.667 \pm 0.333^{b c}$ \\
\hline Juj. & $39.667 \pm 0.667$ & $47.3 \pm 4.077$ & $46 \pm 1$ & $47.5 \pm 2.179$ & $46.5 \pm 2.291$ \\
\hline Ilium & $40.333 \pm 0.333$ & $48.5 \pm 3.617$ & $45.667 \pm 1.833$ & $47.333 \pm 2.186$ & $50.667 \pm 4.055$ \\
\hline Cecum & $25.667 \pm 0.333^{c}$ & $32.333 \pm 1.202^{\mathrm{a}}$ & $29 \pm 0^{b}$ & $29.333 \pm 1.453^{\mathrm{ab}}$ & $30.067 \pm 0.968^{\mathrm{ab}}$ \\
\hline Colon & $4.7 \pm 0.1$ & $5.5 \pm 0.173$ & $4.933 \pm 0.567$ & $4.667 \pm 0.167$ & $7.6 \pm 2.201$ \\
\hline Rectum & $3.333 \pm 0.167$ & $5 \pm 1$ & $4.4 \pm 0.379$ & $4.4 \pm 0.666$ & $2.933 \pm 0.067$ \\
\hline Total & $162.867 \pm 1.593$ & $196.7 \pm 11.663$ & $187.433 \pm 2.714$ & $189.067 \pm 8.02$ & $197.467 \pm 9.828$ \\
\hline
\end{tabular}

Mean values with common superscript in row differ significantly $(\mathrm{p}<0.01)$

Table 4. Relative length (\%) of females GIT parts than total GIT length of partial uropygialectomy treatments.

\begin{tabular}{|c|c|c|c|c|c|}
\hline \multirow[b]{2}{*}{ GIT parts } & \multicolumn{4}{|c|}{ Treatments } & \multirow[b]{2}{*}{ T5 } \\
\hline & T1 (Control) & $\mathrm{T} 2$ & T3 & $\mathrm{T} 4$ & \\
\hline Eso. & $9.625 \pm 0.295$ & $8.559 \pm 0.435$ & $9.603 \pm 0.266$ & $9.697 \pm 0.205$ & $9.72 \pm 0.484$ \\
\hline Pro. V. & $2.245 \pm 0.219$ & $1.903 \pm 0.052$ & $1.706 \pm 0.037$ & $1.772 \pm 0.135$ & $1.748 \pm 0.132$ \\
\hline Giz. & $0.921 \pm 0.009$ & $0.96 \pm 0.094$ & $1.519 \pm 0.342$ & $0.796 \pm 0.035$ & $1.576 \pm 0.286$ \\
\hline Deu. & $12.471 \pm 0.692$ & $12.72 \pm 0.363$ & $12.723 \pm 0.349$ & $12.601 \pm 0.392$ & $12.75 \pm 0.525$ \\
\hline pancreas & $4.913 \pm 0.048^{\mathrm{b}}$ & $5.442 \pm 0.171^{\mathrm{a}}$ & $5.103 \pm 0.121^{\mathrm{ab}}$ & $4.672 \pm 0.123^{b c}$ & $4.398 \pm 0.157^{\mathrm{c}}$ \\
\hline Juj. & $24.352 \pm 0.179$ & $23.966 \pm 0.696$ & $24.539 \pm 0.289$ & $25.135 \pm 0.688$ & $23.669 \pm 1.679$ \\
\hline Ilium & $24.765 \pm 0.056$ & $24.622 \pm 0.562$ & $24.35 \pm 0.703$ & $25.033 \pm 0.413$ & $25.631 \pm 1.35$ \\
\hline Cecum & $15.76 \pm 0.158$ & $16.493 \pm 0.544$ & $15.479 \pm 0.224$ & $15.511 \pm 0.289$ & $15.254 \pm 0.323$ \\
\hline Colon & $2.887 \pm 0.089$ & $2.82 \pm 0.21$ & $2.633 \pm 0.306$ & $2.472 \pm 0.078$ & $3.763 \pm 0.889$ \\
\hline Rectum & $2.049 \pm 0.121$ & $2.516 \pm 0.388$ & $2.345 \pm 0.185$ & $2.311 \pm 0.269$ & $1.49 \pm 0.054$ \\
\hline
\end{tabular}

Mean values with common superscript in row differ significantly $(\mathrm{p}<0.01)$

Table 5. Mean $( \pm$ S.E.) males GIT parts weight $(\mathrm{g})$ of partial uropygialectomy treatments.

\begin{tabular}{|c|c|c|c|c|c|}
\hline \multicolumn{6}{|c|}{ Treatments } \\
\hline GIT parts & T1 (Control) & $\mathrm{T} 2$ & T3 & $\mathrm{T} 4$ & T5 \\
\hline Eso. & $6.833 \pm 0.167^{\mathrm{c}}$ & $10.5 \pm 0.866^{\mathrm{a}}$ & $9.333 \pm 0.882^{\mathrm{ab}}$ & $8 \pm 0^{\mathrm{bc}}$ & $9.667 \pm 0.333^{\mathrm{ab}}$ \\
\hline Pro. V. & $5.333 \pm 0.333$ & $6.333 \pm 0.882$ & $6.333 \pm 1.333$ & $5.333 \pm 0.333$ & $7 \pm 1$ \\
\hline Giz. & $19 \pm 1.528$ & $26 \pm 1.732$ & $26 \pm 0.577$ & $22.667 \pm 3.333$ & $28 \pm 1.155$ \\
\hline Deu. & $5.333 \pm 0.882^{b}$ & $8.333 \pm 1.453^{\mathrm{ab}}$ & $7 \pm 0.577^{b}$ & $6.333 \pm 0.333^{b}$ & $10.333 \pm 0.882^{\circ}$ \\
\hline Pancreas & $1.333 \pm 0.333^{\mathrm{c}}$ & $3 \pm 0^{\mathrm{b}}$ & $2.167 \pm 0.167^{\mathrm{bc}}$ & $2 \pm 0.577^{\mathrm{bc}}$ & $9.167 \pm 0.601^{\mathrm{a}}$ \\
\hline Juj. & $6.333 \pm 0.667^{\mathrm{c}}$ & $13 \pm 1.732^{\mathrm{ab}}$ & $9.333 \pm 0.667^{b c}$ & $9.333 \pm 1.667^{\mathrm{bc}}$ & $14.333 \pm 0.882^{\circ}$ \\
\hline Ilium & $6.333 \pm 0.333^{c}$ & $11 \pm 1^{\mathrm{a}}$ & $9.333 \pm 0.333^{\mathrm{ab}}$ & $7.333 \pm 0.882^{b c}$ & $12 \pm 1.155^{\mathrm{a}}$ \\
\hline Cecum & $3.667 \pm 0.333^{\mathrm{c}}$ & $5.333 \pm 0.333^{\mathrm{a}}$ & $4.667 \pm 0.333^{\mathrm{ab}}$ & $4 \pm 0^{\mathrm{bc}}$ & $5.333 \pm 0.333^{\mathrm{a}}$ \\
\hline Colon & $1 \pm 0.667$ & $1.667 \pm 0.333$ & $1.667 \pm 0.333$ & $1.333 \pm 0.441$ & $2.833 \pm 0.228$ \\
\hline Rectum & $2.333 \pm 0.333$ & $5 \pm 1.528$ & $3.833 \pm 0.167$ & $3.333 \pm 0.333$ & $4.167 \pm 0.167$ \\
\hline Total & $57.5 \pm 0.764^{\mathrm{d}}$ & $90.167 \pm 6.772^{\mathrm{ab}}$ & $79.667 \pm 4.177^{\mathrm{bc}}$ & $69.667 \pm 2.728^{\mathrm{cd}}$ & $102.833 \pm 3.42^{\circ}$ \\
\hline
\end{tabular}

Mean values with common superscript in row differ significantly $(\mathrm{p}<0.01)$

Males in T5 treatment exceeded other treatments $(\mathrm{p}<0.01)$ in the weight of duodenum, pancreas, jejunum, ilium and cecum and the values were 10.333, $9.167,14.333,12$ and 5.333 gm respectively. All the males of PU treatments have outperformed the control group in the total GIT weight property and the highest value was in the fifth treatment $(102.833 \mathrm{gm})$ and followed by T2 (90.167 gm), T3 (79.667 gm), T4 (69.667 gm) and control group (57.5 gm).
In terms of the males' relative weight of digestive systems parts compared to its integral weight (Table 6 ) present that only the relative weights of esophagus and pancreas obtained significant differences $(p<0.01)$ between the transactions for the benefit of the fifth treatment and the values were 9.4 and 8.964 respectively. Table 7 also revealed the superiority of T5 treatment in the males' relative weight than the total live body weight in all of pancreas, jejunum and 
ilium weights. And their values were $0.559,0.874$ and 0.732 in the same order.

Table 8 shows that the effect of partial ablation of the uropygial gland reflected on four GIT parts weights in females, namely esophagus, duodenum, ilium and cecum weights. The highest values in these traits were in T2 $(9 \mathrm{gm}),(7.167 \mathrm{gm}),(8 \mathrm{gm})$ and (5.333 gm) respectively. Duodenal relative weight than the total GIT weight was $(\mathrm{p}<0.01)$ significant different between treatments (Table 9) and the high value reported in T2 (9.659). While in colon relative weight, the T5 shows significantly higher value $(p<0.01)$ than the rest of the treatments. Live body weights in T2, T4 and T5 treatments were significantly $(\mathrm{p}<0.01)$ higher means values than T3 and control groups. However, females live body weights in $\mathrm{T} 3$ treatment were heavier than those in the control group but not at a significant level. The treatments' live body weights from higher to lower value were: T2 (1189.3 gm), T4 (1187.3 gm), T5 (1182.3 gm), T3 (977.6 gm) and T1 (937.3 gm). Table 10 duodenum was the only part achieved important effect $(p<0.01)$ in the relative weight than the total live body weight. However, no significant different between T2, T4, T5 and T1 also between T4, T5, T1 and $\mathrm{T} 3$ were observed.

\section{GIT Parts Density}

In males, the treatments were significantly different in the density of the following parts: Esophagus, gizzard, duodenum, pancreas, jejunum, ilium and cecum. Nevertheless, in females they were: Esophagus, gizzard, duodenum and rectum.

With regard to the males, Table 11 shows the statistical comparison of all studied GIT parts density. T5 treatment predominated on the rest treatments in the following density parts: Duodenum, pancreas, jejunum, ilium and cecum. Males of PU treatments were outperformed $(p<0.05)$ than the control group's males in esophagus density values. Based on an order of priority, they were as following: T2 (0.505), T5 (0.475), T3 (0.444), T4 (0.372) and T1 (0.371). It is worth mentioning that the males in $\mathrm{T} 5$ and $\mathrm{T} 2$ treatments achieved $(\mathrm{p}<0.01)$ biggest values in the total GIT density compared with the rest of the transactions.

Table 6. Relative weight (\%) of males GIT parts than total GIT weight of partial uropygialectomy treatments.

\begin{tabular}{|c|c|c|c|c|c|}
\hline \multirow[b]{2}{*}{ GIT parts } & \multicolumn{4}{|c|}{ Treatments } & \multirow[b]{2}{*}{ T5 } \\
\hline & T1 (Control) & $\mathrm{T} 2$ & $\mathrm{~T} 3$ & $\mathrm{~T} 4$ & \\
\hline Eso. & $11.883 \pm 0.224^{b}$ & $11.633 \pm 0.217^{b}$ & $11.67 \pm 0.536^{b}$ & $11.518 \pm 0.438^{b}$ & $9.4 \pm 0.018^{\mathrm{a}}$ \\
\hline Pro. V. & $9.264 \pm 0.453$ & $7.073 \pm 1.042$ & $7.824 \pm 1.202$ & $7.643 \pm 0.19$ & $6.787 \pm 0.849$ \\
\hline Giz. & $33.12 \pm 3.029$ & $28.873 \pm 0.454$ & $32.748 \pm 1.153$ & $32.381 \pm 4.198$ & $27.225 \pm 0.585$ \\
\hline Deu. & $9.244 \pm 1.413$ & $9.107 \pm 0.918$ & $8.773 \pm 0.445$ & $9.143 \pm 0.769$ & $10.058 \pm 0.821$ \\
\hline pancreas & $2.305 \pm 0.543^{b}$ & $3.364 \pm 0.244^{b}$ & $2.713 \pm 0.065^{b}$ & $2.834 \pm 0.737^{b}$ & $8.964 \pm 0.839^{\mathrm{a}}$ \\
\hline Juj. & $11.009 \pm 1.129$ & $14.297 \pm 0.92$ & $11.729 \pm 0.742$ & $13.386 \pm 2.353$ & $13.927 \pm 0.595$ \\
\hline Ilium & $11.023 \pm 0.642$ & $12.183 \pm 0.379$ & $11.735 \pm 0.191$ & $10.598 \pm 1.526$ & $11.628 \pm 0.803$ \\
\hline Cecum & $6.369 \pm 0.534$ & $5.93 \pm 0.163$ & $5.871 \pm 0.416$ & $5.759 \pm 0.219$ & $5.188 \pm 0.279$ \\
\hline Colon & $1.74 \pm 0.023$ & $1.768 \pm 0.573$ & $2.079 \pm 0.387$ & $1.93 \pm 0.508$ & $2.768 \pm 0.448$ \\
\hline Rectum & $4.044 \pm 0.52$ & $5.771 \pm 2.119$ & $4.858 \pm 0.441$ & $4.809 \pm 0.559$ & $4.055 \pm 0.142$ \\
\hline
\end{tabular}

Mean values with common superscript in row differ significantly $(\mathrm{p}<0.01)$

Table 7. Relative weight (\%) of males GIT parts than total live body weight of partial uropygialectomy treatments.

\begin{tabular}{|c|c|c|c|c|c|}
\hline \multicolumn{6}{|c|}{ Treatments } \\
\hline GIT parts & T1 (Control) & $\mathrm{T} 2$ & $\mathrm{~T} 3$ & $\mathrm{~T} 4$ & T5 \\
\hline Eso. & $0.492 \pm 0.014$ & $0.628 \pm 0.054$ & $0.585 \pm 0.06$ & $0.507 \pm 0.004$ & $0.59 \pm 0.021$ \\
\hline Pro. V. & $0.383 \pm 0.019$ & $0.379 \pm 0.056$ & $0.397 \pm 0.087$ & $0.338 \pm 0.021$ & $0.428 \pm 0.066$ \\
\hline Giz. & $1.37 \pm 0.128$ & $1.555 \pm 0.11$ & $1.627 \pm 0.044$ & $1.44 \pm 0.22$ & $1.708 \pm 0.066$ \\
\hline Deu. & $0.382 \pm 0.058$ & $0.499 \pm 0.088$ & $0.438 \pm 0.038$ & $0.401 \pm 0.018$ & $0.631 \pm 0.057$ \\
\hline Pancreas & $0.095 \pm 0.022^{b}$ & $0.179 \pm 0.002^{\mathrm{b}}$ & $0.136 \pm 0.011^{\mathrm{b}}$ & $0.126 \pm 0.036^{\mathrm{b}}$ & $0.559 \pm 0.035^{\mathrm{a}}$ \\
\hline Juj. & $0.454 \pm 0.043^{c}$ & $0.778 \pm 0.106^{\mathrm{ab}}$ & $0.585 \pm 0.045^{b c}$ & $0.59 \pm 0.102^{\mathrm{bc}}$ & $0.874 \pm 0.051^{\mathrm{a}}$ \\
\hline Ilium & $0.456 \pm 0.03^{b}$ & $0.658 \pm 0.06^{\mathrm{a}}$ & $0.584 \pm 0.025^{\mathrm{ab}}$ & $0.464 \pm 0.052^{\mathrm{b}}$ & $0.732 \pm 0.073^{\mathrm{a}}$ \\
\hline Cecum & $0.264 \pm 0.024$ & $0.319 \pm 0.02$ & $0.292 \pm 0.021$ & $0.254 \pm 0.002$ & $0.325 \pm 0.019$ \\
\hline Colon & $0.072 \pm 0.001$ & $0.1 \pm 0.04$ & $0.105 \pm 0.021$ & $0.085 \pm 0.022$ & $0.173 \pm 0.026$ \\
\hline Rectum & $0.167 \pm 0.022$ & $0.297 \pm 0.088$ & $0.24 \pm 0.009$ & $0.212 \pm 0.023$ & $0.254 \pm 0.012$ \\
\hline Total & $4.135 \pm 0.04^{\mathrm{c}}$ & $5.393 \pm 0.42^{\mathrm{b}}$ & $4.989 \pm 0.298^{b c}$ & $4.417 \pm 0.182^{\mathrm{c}}$ & $6.273 \pm 0.215^{\mathrm{a}}$ \\
\hline
\end{tabular}

Mean values with common superscript in row differ significantly $(\mathrm{p}<0.01)$ 


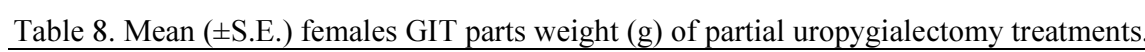

\begin{tabular}{|c|c|c|c|c|c|}
\hline \multirow[b]{2}{*}{ GIT parts } & \multicolumn{4}{|c|}{ Treatments } & \multirow{2}{*}{$\mathrm{T} 5$} \\
\hline & T1 (Control) & $\mathrm{T} 2$ & $\mathrm{~T} 3$ & $\mathrm{~T} 4$ & \\
\hline Eso. & $6.667 \pm 0.167^{b}$ & $9 \pm 1^{\mathrm{a}}$ & $5.333 \pm 0.667^{b}$ & $7.333 \pm 0.667^{\mathrm{ab}}$ & $7 \pm 0.577^{\mathrm{ab}}$ \\
\hline Pro. V. & $4 \pm 0$ & $5.667 \pm 0.333$ & $4 \pm 0.577$ & $4.333 \pm 0.333$ & $5.333 \pm 0.882$ \\
\hline Giz. & $19 \pm 0$ & $22 \pm 1.155$ & $18.667 \pm 0.333$ & $21 \pm 1$ & $2.333 \pm 5.457$ \\
\hline Deu. & $4.667 \pm 0.333^{b c}$ & $7.167 \pm 0.441^{\mathrm{a}}$ & $3.667 \pm 0.333^{c}$ & $6 \pm 0.577^{\mathrm{ab}}$ & $6 \pm 0.577^{\mathrm{ab}}$ \\
\hline pancreas & $1.667 \pm 0.333$ & $3 \pm 0$ & $1.5 \pm 0.289$ & $2.333 \pm 0.333$ & $2.833 \pm 0.601$ \\
\hline Juj. & $8.333 \pm 0.667$ & $9.667 \pm 1.202$ & $8 \pm 0.577$ & $8.667 \pm 0.333$ & $8.333 \pm 1.202$ \\
\hline Ilium & $5.667 \pm 0.333^{b}$ & $8 \pm 0.577^{\mathrm{a}}$ & $6.333 \pm 0.333^{\mathrm{ab}}$ & $6 \pm 0.577^{\mathrm{ab}}$ & $8 \pm 1^{\mathrm{a}}$ \\
\hline Cecum & $3 \pm 0^{c}$ & $5.333 \pm 0.333^{\mathrm{a}}$ & $3.333 \pm 0.333^{b c}$ & $3.333 \pm 0.333^{b c}$ & $4.667 \pm 0882^{\mathrm{ab}}$ \\
\hline Colon & $1 \pm 0$ & $1.267 \pm 0.371$ & $1.1 \pm 0.208$ & $1 \pm 0$ & $2.167 \pm 0.441$ \\
\hline Rectum & $2 \pm 0$ & $3 \pm 0.577$ & $3 \pm 0$ & $3 \pm 0.577$ & $3.433 \pm 0.233$ \\
\hline Total & $56 \pm 1.5$ & $74.1 \pm 3.121$ & $54.933 \pm 0.233$ & $63 \pm 1.528$ & $70.1 \pm 10.027$ \\
\hline
\end{tabular}

Mean values with common superscript in row differ significantly $(\mathrm{p}<0.01)$

Table 9. Relative weight (\%) of females GIT parts than total GIT weight of partial uropygialectomy treatments.

\begin{tabular}{|c|c|c|c|c|c|}
\hline \multirow[b]{2}{*}{ GIT parts } & \multicolumn{4}{|c|}{ Treatments } & \multirow[b]{2}{*}{ T5 } \\
\hline & T1 (Control) & $\mathrm{T} 2$ & $\mathrm{~T} 3$ & $\mathrm{~T} 4$ & \\
\hline Eso. & $11.939 \pm 0.634$ & $12.08 \pm 0.936$ & $9.711 \pm 1.22$ & $11.603 \pm 0.803$ & $10.176 \pm 0.676$ \\
\hline Pro. V. & $7.153 \pm 0.197$ & $7.682 \pm 0.613$ & $7.266 \pm 1.058$ & $6.878 \pm 0.49$ & $7.579 \pm 0.292$ \\
\hline Giz. & $33.979 \pm 0.935$ & $29.842 \pm 2.264$ & $33.986 \pm 0.724$ & $33.451 \pm 2.445$ & $31.124 \pm 3.448$ \\
\hline Deu. & $8.313 \pm 0.383^{\mathrm{ab}}$ & $9.659 \pm 0.24^{\mathrm{a}}$ & $6.67 \pm 0.583^{b}$ & $9.493 \pm 0.706^{\mathrm{a}}$ & $8.772 \pm 0.942^{\mathrm{a}}$ \\
\hline pancreas & $2.948 \pm 0.53$ & $4.063 \pm 0.175$ & $2.733 \pm 0.535$ & $3.691 \pm 0.466$ & $4.11 \pm 0.81$ \\
\hline Juj. & $14.838 \pm 0.815$ & $13.011 \pm 1.308$ & $14.558 \pm 1.017$ & $13.79 \pm 0.789$ & $11.881 \pm 0.398$ \\
\hline Ilium & $10.101 \pm 0.334$ & $10.769 \pm 0.334$ & $11.525 \pm 0.567$ & $9.493 \pm 0.706$ & $11.498 \pm 0.673$ \\
\hline Cecum & $5.365 \pm 0.148$ & $7.194 \pm 0.275$ & $6.064 \pm 0.585$ & $5.28 \pm 0.446$ & $6.645 \pm 0.903$ \\
\hline Colon & $1.788 \pm 0.049^{b}$ & $1.701 \pm 0.483^{b}$ & $2.006 \pm 0.388^{b}$ & $1.589 \pm 0.039^{b}$ & $3.044 \pm 0.202^{\mathrm{a}}$ \\
\hline Rectum & $3.577 \pm 0.098$ & $3.997 \pm 0.616$ & $5.461 \pm 0.023$ & $4.733 \pm 0.844$ & $5.171 \pm 1.049$ \\
\hline
\end{tabular}

Mean values with common superscript in row differ significantly $(\mathrm{p}<0.01)$

Table 10. Relative weight (\%) of females GIT parts than total live body weight of partial uropygialectomy treatments.

\begin{tabular}{|c|c|c|c|c|c|}
\hline \multirow[b]{2}{*}{ GIT parts } & \multicolumn{4}{|c|}{ Treatments } & \multirow[b]{2}{*}{$\mathrm{T} 5$} \\
\hline & T1 (Control) & $\mathrm{T} 2$ & $\mathrm{~T} 3$ & $\mathrm{~T} 4$ & \\
\hline Eso. & $0.713 \pm 0.034$ & $0.759 \pm 0.091$ & $0.548 \pm 0.076$ & $0.619 \pm 0.064$ & $0.592 \pm 0.046$ \\
\hline Pro. V. & $0.427 \pm 0.01$ & $0.476 \pm 0.024$ & $0.407 \pm 0.052$ & $0.365 \pm 0.029$ & $0.451 \pm 0.073$ \\
\hline Giz. & $2.029 \pm 0.048$ & $1.851 \pm 0.111$ & $1.909 \pm 0.017$ & $1.767 \pm 0.062$ & $1.887 \pm 0.459$ \\
\hline Deu. & $0.497 \pm 0.027^{\mathrm{ab}}$ & $0.603 \pm 0.04^{\mathrm{a}}$ & $0.375 \pm 0.035^{\mathrm{b}}$ & $0.507 \pm 0.056^{\mathrm{ab}}$ & $0.506 \pm 0.042^{\mathrm{ab}}$ \\
\hline pancreas & $0.177 \pm 0.033$ & $0.252 \pm 0.003$ & $0.154 \pm 0.03$ & $0.197 \pm 0.031$ & $0.238 \pm 0.048$ \\
\hline Juj. & $0.887 \pm 0.055$ & $0.812 \pm 0.1$ & $0.818 \pm 0.053$ & $0.73 \pm 0.02$ & $0.703 \pm 0.097$ \\
\hline Ilium & $0.604 \pm 0.025$ & $0.673 \pm 0.052$ & $0.649 \pm 0.045$ & $0.507 \pm 0.056$ & $0.675 \pm 0.078$ \\
\hline Cecum & $0.32 \pm 0.008$ & $0.449 \pm 0.028$ & $0.342 \pm 0.04$ & $0.282 \pm 0.032$ & $0.393 \pm 0.07$ \\
\hline Colon & $0.107 \pm 0.003$ & $0.107 \pm 0.033$ & $0.112 \pm 0.021$ & $0.084 \pm 0.001$ & $0.183 \pm 0.037$ \\
\hline Rectum & $0.214 \pm 0.005$ & $0.253 \pm 0.05$ & $0.307 \pm 0.006$ & $0.253 \pm 0.05$ & $0.291 \pm 0.023$ \\
\hline Total & $5.974 \pm 0.078$ & $6.236 \pm 0.311$ & $5.623 \pm 0.113$ & $5.312 \pm 0.204$ & $5.919 \pm 0.819$ \\
\hline
\end{tabular}

Mean values with common superscript in row differ significantly $(\mathrm{p}<0.01)$

The densities of female digestive system in Table 12 shows that $\mathrm{T} 2$ treatments achieved to $(\mathrm{p}<0.05)$ consequentially exceed in the density of the esophagus. The values according to the sequence of priorities were as following: T2 (0.538), T1 (0.426), T4 (0.399), T5 $(0.368)$ and $\mathrm{T} 3(0.295)$. No significant difference was indicated between $\mathrm{T} 3$ and $\mathrm{T} 4$ also between T4, T5 and T3. Females' gizzards were more density in T4, T1 and
T2 treatments than those in the T5 and T3 at a significant level $(\mathrm{p}<0.01)$. The duodenums' densities of treatments from higher to lower value were: T2 (0.289), T4 (0.251), T5 (0.241), T1 (0.23) and T3 (0.154). Furthermore, the females' rectum densities in PU treatments were morally $(\mathrm{p}<0.01)$ higher than females within the control group as following: T5 (1.168), T4 (0.699), T3 (0.0692), T2 (0.607) and T1 (0.603). 
Table 11. Mean $( \pm$ S.E. $)$ males GIT parts density $(\mathrm{g} / \mathrm{cm})$ of partial uropygialectomy treatments.

\begin{tabular}{|c|c|c|c|c|c|}
\hline \multirow[b]{2}{*}{ GIT parts } & \multicolumn{4}{|c|}{ Treatments } & \multirow[b]{2}{*}{$\mathrm{T} 5$} \\
\hline & T1 (Control) & $\mathrm{T} 2$ & T3 & $\mathrm{T} 4$ & \\
\hline Eso. & $0.371 \pm 0.021^{\mathrm{b}}$ & $0.505 \pm 0.04^{\mathrm{a}}$ & $0.444 \pm 0.04^{\mathrm{ab}}$ & $0.372 \pm 0.005^{\mathrm{b}}$ & $0.475 \pm 0.014^{\mathrm{a}}$ \\
\hline Pro. V. & $1.832 \pm 0.292$ & $1.563 \pm 0.176$ & $1.643 \pm 0.308$ & $1.603 \pm 0.088$ & $1.764 \pm 0.124$ \\
\hline Giz. & $12.667 \pm 1.018^{\mathrm{a}}$ & $11.311 \pm 0.241^{\mathrm{a}}$ & $12.772 \pm 1.963^{\mathrm{a}}$ & $11.804 \pm 1.196^{\mathrm{a}}$ & $5.923 \pm 0.524^{b}$ \\
\hline Deu. & $0.247 \pm 0.052^{\mathrm{b}}$ & $0.322 \pm 0.066^{\mathrm{ab}}$ & $0.28 \pm 0.021^{\mathrm{b}}$ & $0.255 \pm 0.003^{\mathrm{b}}$ & $0.442 \pm 0.32^{\mathrm{a}}$ \\
\hline Pancreas & $0.148 \pm 0.037^{\mathrm{b}}$ & $0.293 \pm 0.007^{\mathrm{b}}$ & $0.201 \pm 0.018^{\mathrm{b}}$ & $0.194 \pm 0.055^{\mathrm{b}}$ & $1.019 \pm 0.067^{\mathrm{a}}$ \\
\hline Juj. & $0.146 \pm 0.02^{\mathrm{b}}$ & $0.246 \pm 0.022^{\mathrm{a}}$ & $0.193 \pm 0.019^{\mathrm{ab}}$ & $0.202 \pm 0.035^{\mathrm{ab}}$ & $0.262 \pm 0.023^{\mathrm{a}}$ \\
\hline Ilium & $0.131 \pm 0.014^{b}$ & $0.193 \pm 0.009^{\mathrm{a}}$ & $0.187 \pm 0.021^{\mathrm{ab}}$ & $0.136 \pm 0.014^{\mathrm{b}}$ & $0.199 \pm 0.024^{\mathrm{a}}$ \\
\hline Cicum & $0.127 \pm 0.012^{\mathrm{b}}$ & $0.162 \pm 0.013^{\mathrm{a}}$ & $0.152 \pm 0.004^{\mathrm{ab}}$ & $0.121 \pm 0.004^{b}$ & $0.172 \pm 0.014^{\mathrm{a}}$ \\
\hline Colon & $0.195 \pm 0.013$ & $0.246 \pm 0.092$ & $0.273 \pm 0.054$ & $0.212 \pm 0.031$ & $0.34 \pm 0.045$ \\
\hline Rectum & $0.607 \pm 0.074$ & $1.141 \pm 0.173$ & $0.936 \pm 0.136$ & $0.765 \pm 0.131$ & $1 \pm 0$ \\
\hline Total & $0.312 \pm 0.007^{\mathrm{d}}$ & $0.416 \pm 0.026^{\mathrm{ab}}$ & $0.392 \pm 0.029^{\mathrm{bc}}$ & $0.339 \pm 0.009^{\mathrm{cd}}$ & $0.466 \pm 0.022^{\mathrm{a}}$ \\
\hline
\end{tabular}

Mean values with common superscript in row differ significantly $(\mathrm{p}<0.05)$

Mean values of pancreas density and total GIT density rows differ significantly $(p<0.01)$

Table 12 . Mean $( \pm$ S.E.) females GIT parts density $(\mathrm{g} / \mathrm{cm})$ of partial uropygialectomy treatments.

\begin{tabular}{|c|c|c|c|c|c|}
\hline \multirow[b]{2}{*}{ GIT parts } & \multicolumn{4}{|c|}{ Treatments } & \multirow[b]{2}{*}{$\mathrm{T} 5$} \\
\hline & T1 (Control) & $\mathrm{T} 2$ & $\mathrm{~T} 3$ & $\mathrm{~T} 4$ & \\
\hline Eso. & $0.426 \pm 0.01^{\mathrm{ab}}$ & $0.538 \pm 0.061^{\mathrm{a}}$ & $0.295 \pm 0.03^{\mathrm{c}}$ & $0.399 \pm 0.026^{\mathrm{bc}}$ & $0.368 \pm 0.038^{b c}$ \\
\hline Pro. V. & $1.111 \pm 0.111$ & $1.517 \pm 0.056$ & $1.26 \pm 0.215$ & $1.317 \pm 0.175$ & $1.579 \pm 0.309$ \\
\hline Giz. & $12.667 \pm 0^{\mathrm{a}}$ & $11.778 \pm 0.339^{\mathrm{a}}$ & $7.117 \pm 1.25^{\mathrm{b}}$ & $14 \pm 0.667^{\mathrm{a}}$ & $7.278 \pm 1.299^{\mathrm{b}}$ \\
\hline Deu. & $0.23 \pm 0.016^{\mathrm{a}}$ & $0.289 \pm 0.026^{\mathrm{a}}$ & $0.154 \pm 0.015^{b}$ & $0.251 \pm 0.012^{\mathrm{a}}$ & $0.241 \pm 0.032^{\mathrm{a}}$ \\
\hline Pancreas & $0.208 \pm 0.042$ & $0.282 \pm 0.009$ & $0.156 \pm 0.028$ & $0.265 \pm 0.036$ & $0.333 \pm 0.085$ \\
\hline Juj. & $0.21 \pm 0.016$ & $0.204 \pm 0.013$ & $0.175 \pm 0.016$ & $0.183 \pm 0.012$ & $0.182 \pm 0.032$ \\
\hline Ilium & $0.14 \pm 0.008$ & $0.166 \pm 0.012$ & $0.139 \pm 0.006$ & $0.126 \pm 0.008$ & $0.157 \pm 0.013$ \\
\hline Cicum & $0.117 \pm 0.002$ & $0.165 \pm 0.01$ & $0.115 \pm 0.011$ & $0.113 \pm 0.006$ & $0.155 \pm 0.029$ \\
\hline Colon & $0.213 \pm 0.005$ & $0.231 \pm 0.068$ & $0.222 \pm 0.027$ & $0.215 \pm 0.007$ & $0.3 \pm 0.039$ \\
\hline Rectum & $0.603 \pm 0.032^{\mathrm{b}}$ & $0.607 \pm 0.074^{\mathrm{b}}$ & $0.692 \pm 0.062^{\mathrm{b}}$ & $0.699 \pm 0.151^{\mathrm{b}}$ & $1.168 \pm 0.056^{\mathrm{a}}$ \\
\hline Total & $0.344 \pm 0.008$ & $0.379 \pm 0.027$ & $0.293 \pm 0.005$ & $0.334 \pm 0.006$ & $0.353 \pm 0.041$ \\
\hline
\end{tabular}

Mean values of esophagus and duodenum density rows differ significantly $(\mathrm{p}<0.05)$

Mean values of gizzard and rectal density rows differ significantly $(\mathrm{p}<0.01)$

\section{Discussion}

Present research observed that the uropygial gland located on the base of the tail, dorsally to the levator caudal muscle. It can be evidenced by palpation above the last sacral vertebra and the first caudle vertebra. These results are coincident with those reported by (Nickel et al., 1977; Montalti and Saliban, 2000; Gezici, 2002) and pointed out with (Aslan et al., 2000) who reported that the gland is lying on the pygostyl muscle. Uropygial gland in Akar Putra chicken has hart shape with broad bean size. While (Calislar, 1986) mentioned that the gland has chicken egg size in pelican bird and almond bean in ducks. Present experiment found that the uropygial gland contains right and left lobes which have been separated by an inter-lobular barrier except the lobes' adhesion area in the isthmus at the third back of the gland and these observations consistent with (Getty, 1975). Uropygial gland has anuropygial papilla lies dorso-caudally of the gland and hasuropygialwike. The gland canals have a single opening at each lobe and possess a pair of canals. While (Shawkey et al., 2003) stated that the uropygialpapilla in geese was short, wide and held two openings for their canals, in chickens the papilla is long and thin while in turkey, the papilla is wide on the other hands some birds such as Musk duck lacks the uropygialwike.

The results showed that the partial ablation of the uropygial gland did not have any serious consequence for the survival of akarputra chicken and no mortality had happened during the trial period. That agree with (Jacob, 1976; Chen et al., 2003) whom considering the physiological role of the uropygial gland, it appears that the gland is not necessarily present in all groups of birds. This fact, observed in a number of species, together with the lack of a clear-cut ecological correspondence suggests that, when present, the function of the gland may be diverse but not essential. In this regard, it is interesting that the extirpation of the gland was not dangerous on survival of goslings, hens and passerine birds.

The results of present study improve that the removing of the uropygial gland has highly significantly effect on the most of GIT parts characteristics of males and females akar putra chicken. Figure 1 shows that the males of Partial Uropygialectomy (PU) treatments had longer 
esophagus, proventriculus, gizzard, pancreas, jejunum and colon than their control group counterparts and their variation ratios than control values were: $9.9-16.2 \%, 11.1$ $34.4 \%, 26.7-220 \%, 0-20.4 \%, 4.9-26.1$ and $18.1-60.6$ respectively. Whereas, females of PU treatments as shown in Fig. 2 had longer esophagus, pancreas and cecum compared with females in control and their variation ratio were $6.8-22.3 \%, 8.3-33.3 \%$ and $13-26 \%$. Figure 3 presents that total GIT weight were 21.2 to $78.8 \%$ heavier in males of PU treatments, mainly in the esophagus, duodenum, pancreas, jejunum, ilium and cecum. However, no significant impact $(\mathrm{p}>0.05)$ between treatments was recorded in the total females GIT weight; moreover, females of PU treatments had 5.9-41.2\% heavier ilium and $11.1-77.8 \%$ heavier cecum as shown in
Fig. 4. However, the variation ratio of the total females GIT parts densities were: T2 (10.174), T3 (-14.82), T4 $(-2.907)$ and T5 $(2,616)$ as presented in Fig. 6 , but these variances were not in significant level $(p>0.05)$ between treatments. Nevertheless, the total GIT parts density was highly different $(p<0.01)$ for males in PU treatments and their variation ratio based on Fig. 5 were: T2 (33.333), T3 (25.641), T4 (8.654) and T5 (49.359). These results agree with (Al-Hassani et al., 2008) when they tested the effect of removing the uropygialgland (uropygialectomy) on same semen trails of broiler breeder males and they got highly significantly effects of removing the gland on all semen tested trails. Furthermore, they recommended that uropygialectomy could be used as a tool to improve fertility in the broiler breeders aged between 38-54 weeks.

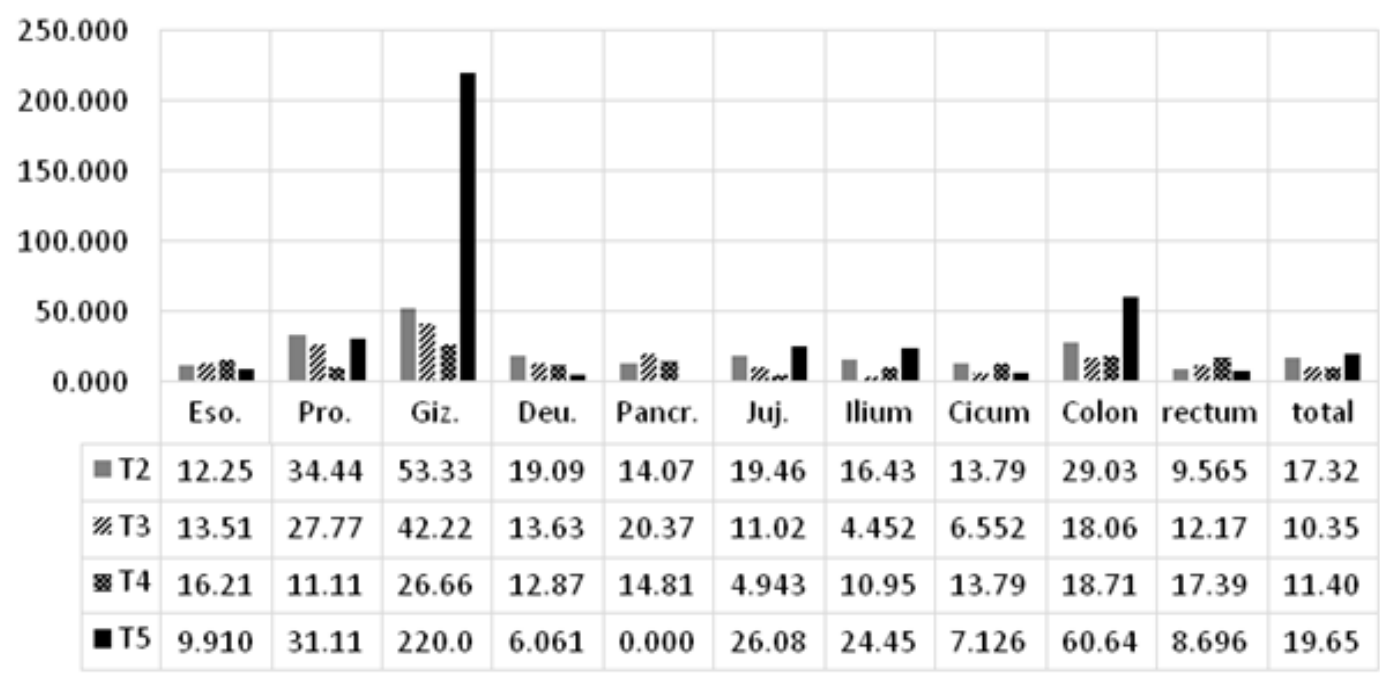

Fig. 1. Variation ratio (\%) of males' GIT parts length of PU treatments than control group

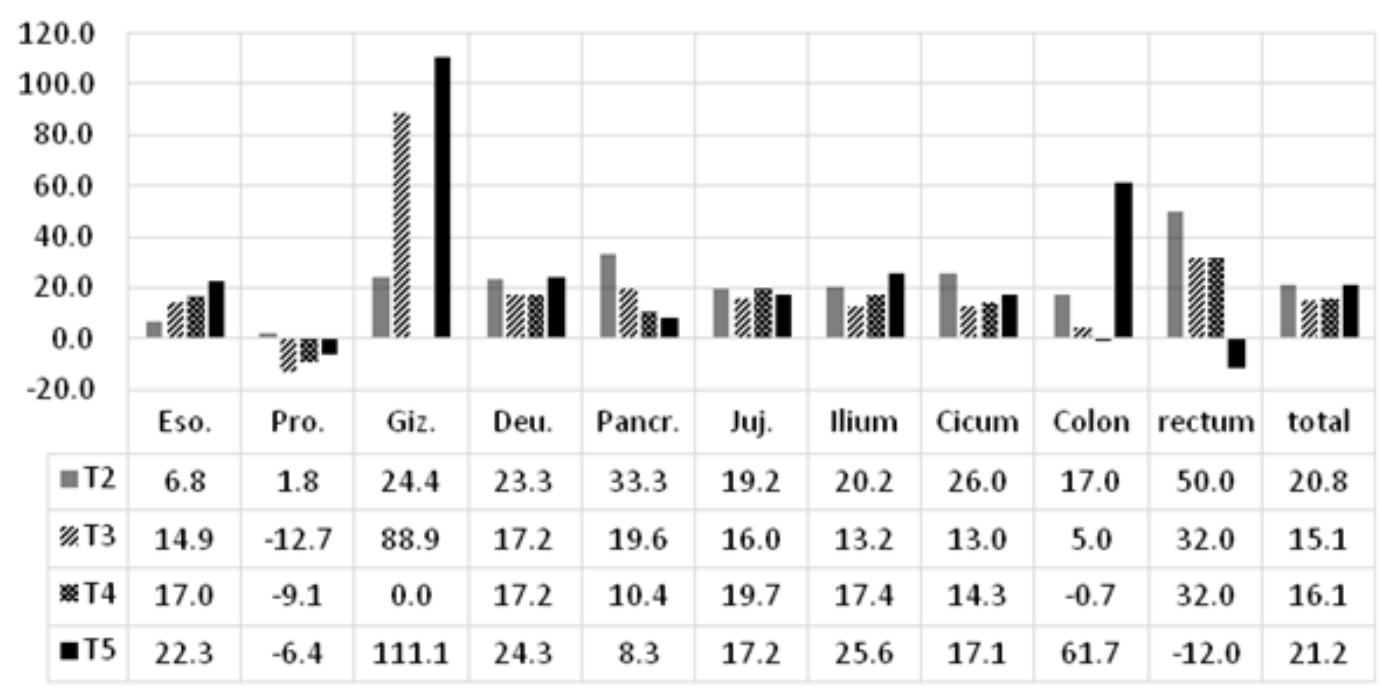

Fig. 2. Variation ratio (\%) of females' GIT parts length of PU treatments than control group 


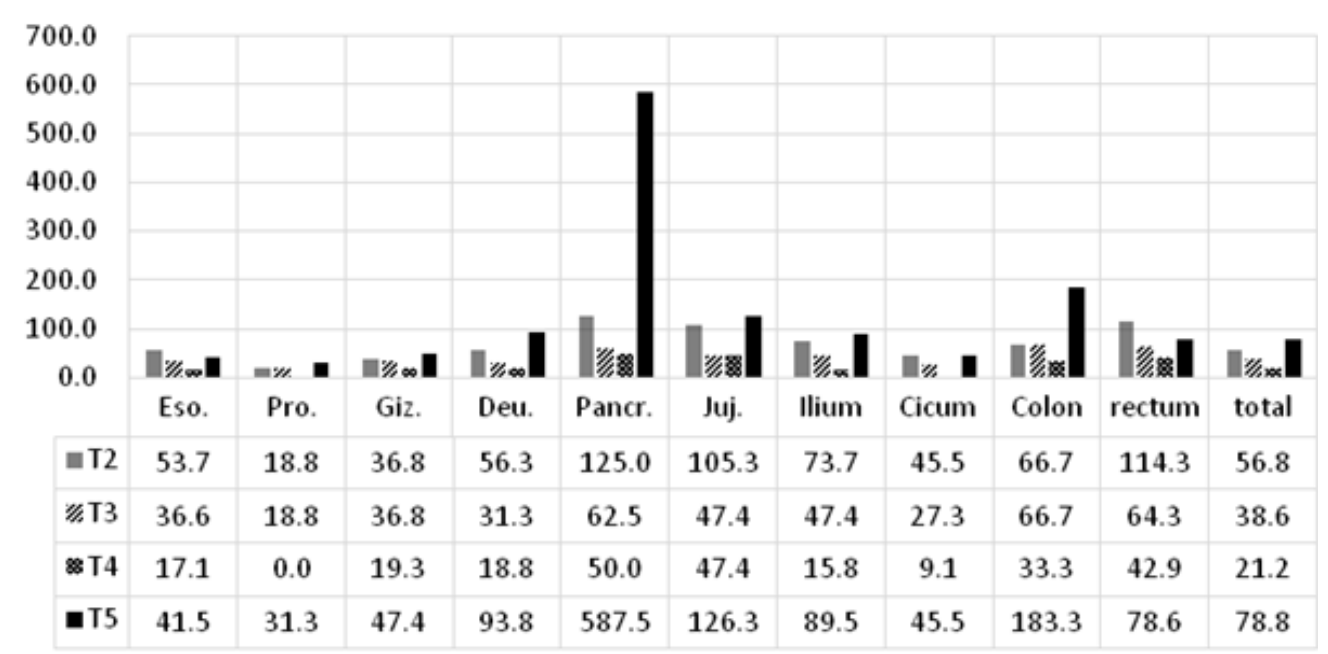

Fig. 3. Variation ratio (\%) of males' GIT parts weight of PU treatments than control group

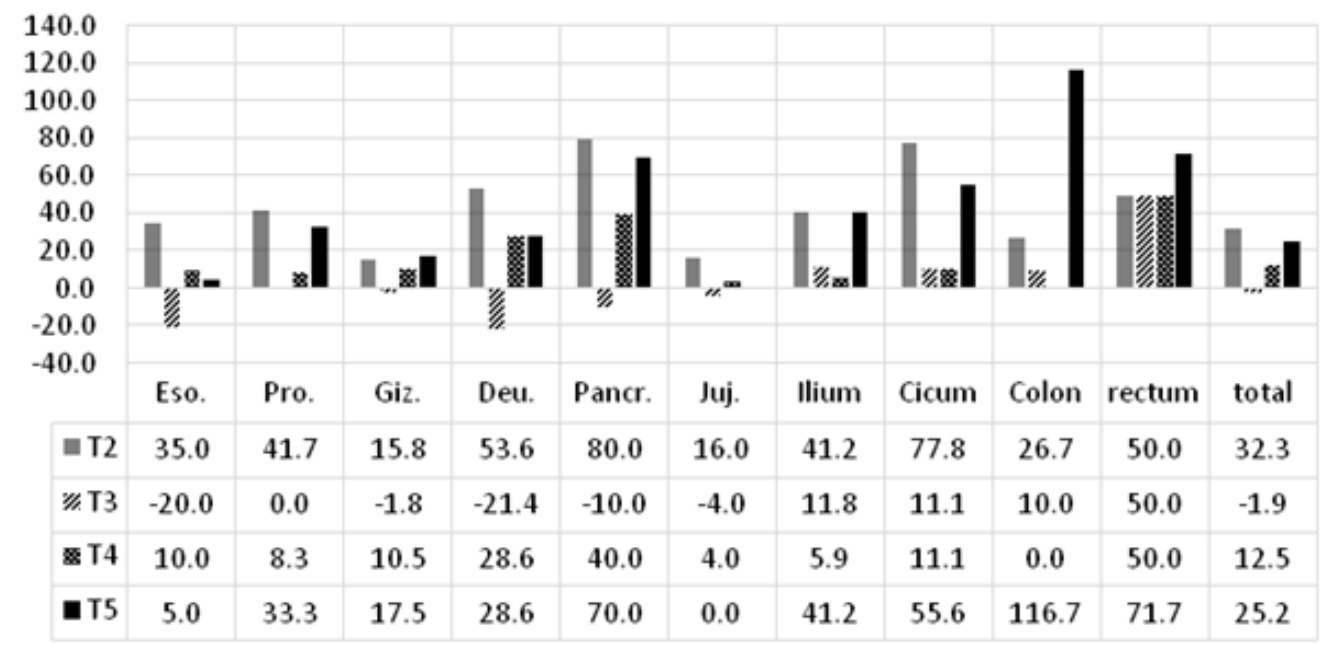

Fig. 4. Variation ratio (\%) of females' GIT parts weight of PU treatments than control group

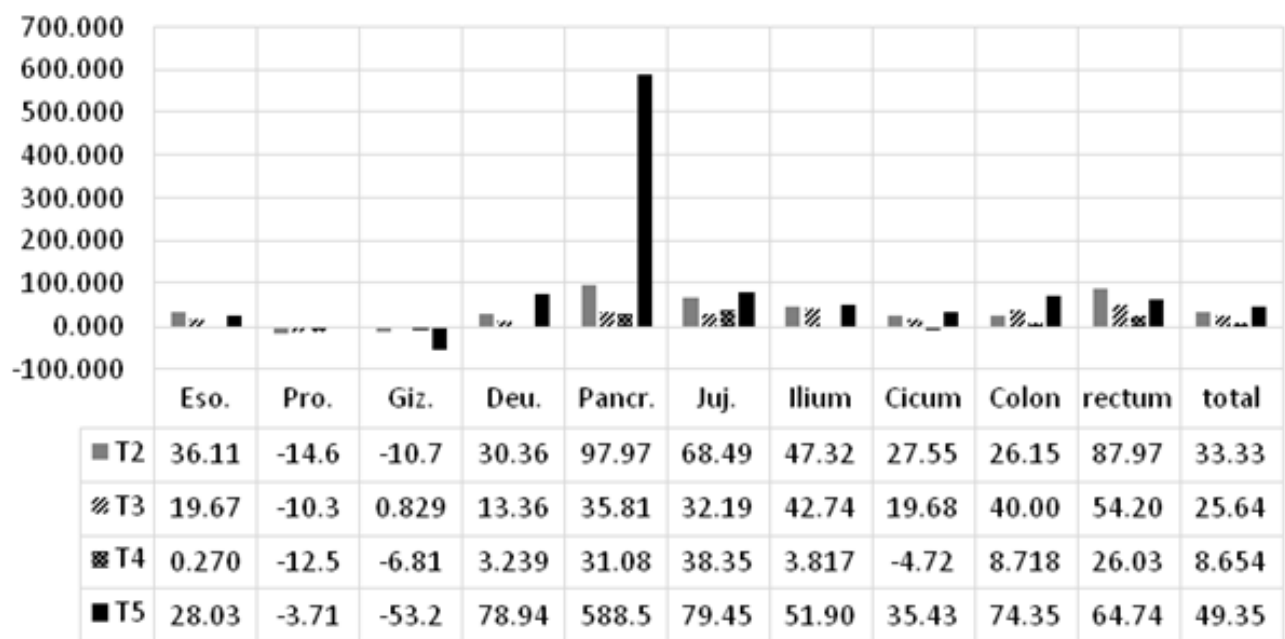

Fig. 5. Variation ratio (\%) of males' GIT parts density of PU treatments than control group 


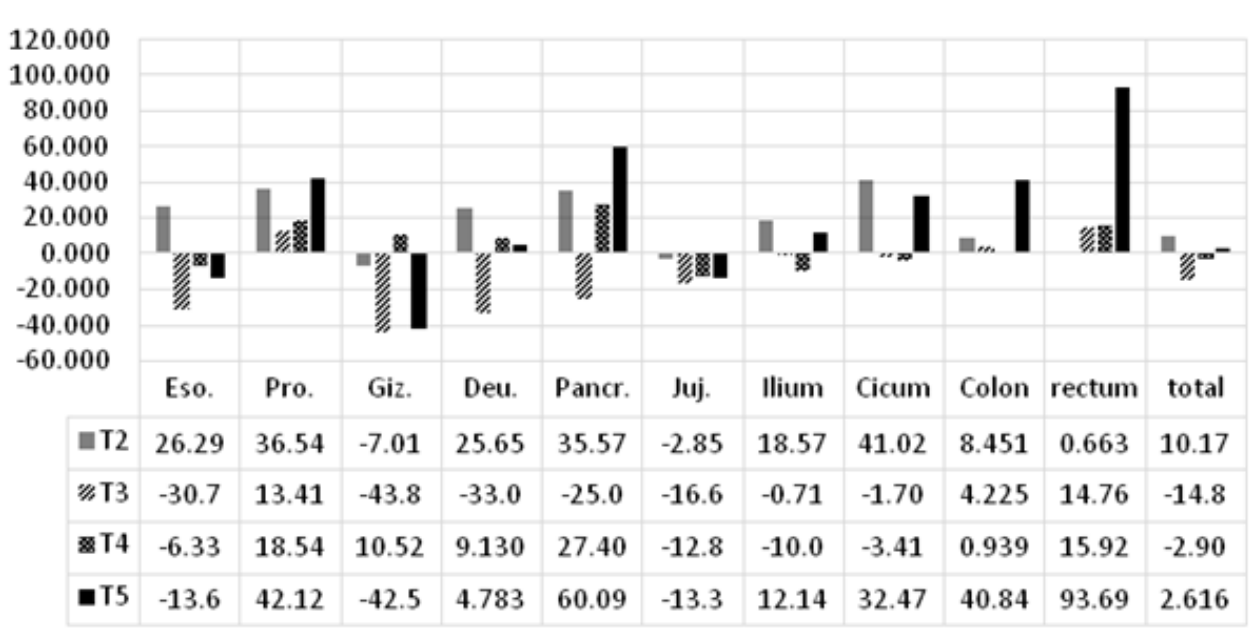

Fig. 6. Variation ratio (\%) of females' GIT parts density of PU treatments than control group

Some previous studies have touched on the study of morphometrical measurements of the digestive system in some birds, such as chickens, ducks, geese, pigeons and turkeys (Rosenberg, 1941; Hodges, 1975; Muelling and Buda, 2002). These studies have indicated that the length range of the duodenum was $22-35 \mathrm{~cm}$ in chicken, $40-49 \mathrm{~cm}$ in goose, $22-38 \mathrm{~cm}$ in duck, $12-22$ $\mathrm{cm}$ in pigeon and $29-39 \mathrm{~cm}$ in turkey. This observation was similar with Hassouna (2001), where, the author stated that the length of the duodenal loop and its parts as well as its shape and extension varied in birds. Furthermore, the previous studies were recorded that the length range of the jejuno-ileum was $98-138 \mathrm{~cm}$ in chicken, $170-213 \mathrm{~cm}$ in goose, $100-158 \mathrm{~cm}$ in duck, 53$84 \mathrm{~cm}$ in pigeon and $200-250 \mathrm{~cm}$ in turkey. This finding was agreed with Hassouna (2001) who stated that in all bird species, the jejunum was the longest part of the small intestine and the authorfound that lowest mean percentage of the length of the ileum to the total length of the small intestine in chicken $(2.7 \%)$. In terms of caeca range length was $12-25 \mathrm{~cm}$ in chicken, 22-34 $\mathrm{cm}$ in goose, $10-20 \mathrm{~cm}$ in duck and $2-7 \mathrm{~cm}$ in pigeon. These results were similar with Hassouna (2001) who proved that caeca were long cylindrical expansions in chickens. And regarding to the rectum-cloacal range length, the values was $8-11 \mathrm{~cm}$ in chicken, $16-22 \mathrm{~cm}$ in goose, $8-13 \mathrm{~cm}$ in duck and $3-4 \mathrm{~cm}$ in pigeon. Finally, they reported the total GIT length range of the chicken, goose, duck, pigeon and turkey, the values were as fallowing: 152-234, 279-352, 150-250, 72-125 and $390-500 \mathrm{~cm}$ respectively.

\section{Conclusion and Suggestion}

Based on the research result and discussion, it can be concluded that stopping the function of the uropygial gland by partial surgical removal technique caused significant improvement in the morphological characteristics of Akar Putra chicken digestive system.
Morphometrical characteristics of male and female GIT segments 12-week-old measurements demonstrate with impossible doubt that the PU operation has a high and explicit impact on the exploratory birds. The PU operation reflection appears prominently on the live body weight and the length, weight and density of most GIT segments. It is assumed that the oil of the uropygial gland had a positive impact on the body's performance after removing the gland. Based on the conclusion, the research team creates several suggestions, as follows:

- Developing the research about removing the uropygial gland in one week-old chicks so that it can increase the effect of the uropygial gland oil on the body's performance

- Developing the research about the oil of uropygial gland biochemistry so that the mechanism of enhancing the body's performance after removing the gland can be precisely identified

\section{Acknowledgment}

This paper was supported by University of Putra Malaysia in 2015. We thank Prof Saad A. Naji, Poultry Science scientist, Department of Animal Resources, Dean of Agriculture faculty, University of Al-qadisiya (Iraq), for the technical assistance.

\section{Author's Contributions}

Hasan, S.A. Jawad: DESIGNed the study, collected all the data, analysed all the data, advised on data analysis and prepared the paper.

I.H Lokman: Designed the study and prepared the paper.

A.B.Z. Zuki: Designed the study and advised on data analysis. 
A.B. Kassim: Designed the study and advised on data analysis.

\section{Ethics}

This article is original and contains unpublished material. The corresponding author confirms that all of the other authors have read and approved the manuscript and no ethical issues involved.

\section{References}

Al-Hassani, D.H., I.A. Abdul-Hassan and S.A. Naji, 2008. Effect of uropygialectomy on some semen traits of broiler breeder males. Proceedings of the 23rd World's Poultry Congress, (WPC'08), Australia.

Aslan, K., S. Ozcan and I. Kürtül, 2000. Arterial vascularization of the uropygial glands (gl. Uropygialis) in geese (Anser anser) and ducks (Anas platyrhynches). Anatomia, Histologia, Embryol., 29: 291-293. DOI: 10.1046/j.1439-0264.2000.00274.x

Calislar, Т., 1986. Anatomy of domestic animals п. Dissection of Horse and HEN, Faculty of Veterinary Medicine, Istanbul University, Istanbul.

Chen, Y.H., M.J. Kou, F.M. Pan and L.L. Lu, 2003. Effects of uropygial gland removal on the growth performance and plasma characteristics in female white roman goslings from 3 to 10 weeks of age. Tunghai J., 44: 7-13.

de Verdal, H., S. Mignon-Grasteau, C. Jeulin, E. Bihan-Duval and M. Leconte et al., 2010. Digestive tract measurements and histological adaptation in broiler lines divergently selected for digestive efficiency. Poultry Sci., 89: 1955-1961. DOI: $10.3382 /$ ps.2010-813

Duncan, B.D., 1955. Multiple range and multiple F tests. Biometrics, 11: 1-42. DOI: 10.2307/3001478

Gaspers, V., 1991. Teknik Penarikan Contoh Untuk Penelitian Survey. 1st Edn., UPT Perpustakaan Universitas Jember, Tarsito, Bandung, pp: 345.

Genstat, 2003. Genstat 5.0 Release 4.23DE. LawesAgric, Trust, Rothamsted Exp. Stn., UK.

Getty, R., 1975. Anatomy of the Domestic Animals. 5th Edn., Saunders (W.B.) Co Ltd., ISBN-10: 0721641024, pp: 2130

Gezici, M., 2002. Skin and Epithermoidal Features. In: Anatomy of Domestic Birds, Medisan pub-Lishing Company, Turkey, pp: 222-222.

Gonzales, E., J. Buyse, M.M. Loddi, T.S. Takita and N. Buys et al., 1998. Performance, incidence of metabolic disturbances and endocrine variables of food-restricted male broiler chickens. Br. Poultry Sci., 39: 671-678. DOI: 10.1080/00071669888557
Goodwin, D., 1983. Pigeons and Doves of the World. 3rd Edn., British Museum, Ithaca, ISBN-10: 0565008471, pp: 363.

Hassouna, E.M.A., 2001. Some anatomical and morphometrical studies on the intestinal tract of chicken, duck, goose, turkey, pigeon, dove, quail, sparrow, heron, jackdaw, hoopoe, kestrel and owl. Assiut Vet. Med. J., 44: 47-78.

Hodges, R.D., 1975. The Histology of the Fowl. 1st Edn., Academic Press, London, ISBN-10: 0123513502, pp: 648.

Jacob, 1992. Systematics and the analysis of integumental lipids. Bulletin British Ornithological Club, Centenary, 112A: 159-167.

Jacob, 1976. Bird Waxes. In: Chemistry and Biochemistry of Natural Waxes, Kolattukudy, P.E. (Ed.), Elsevier Scientific Pub. Co., Amsterdamp, ISBN-10: 0444414703, pp: 93-146.

Jawad, H.S., L.H.B. Idris, S.A. Naji, M.B. Bakar and A.B. Kassim, 2015. Partial ablation of uropygial gland effect on production performance of Akar Putra chicken. Int. J. Poultry Sci., 14: 213-221.

Johnston, D.W., 1988. A Morphological Atlas of the Avian Uropygial Gland. 1st Edn., British Museum (Natural History), London, ISBN-10: 0565050419, pp: 259.

Julian, R.J., 1997. Causes and prevention of ascites in broilers. Zootec. Int., 4: 52-53.

Julian, R.J., 1998. Rapid growth problems: Ascites and skeletal deformities in broilers. Poultry Sci., 77: 1773-1780. DOI: 10.1093/ps/77.12.1773

Leeson, S. and J.D. Summers, 1997. Commercial Poultry Nutrition. 2nd Edn., University Books, ISBN-10: 0969560028, pp: 353.

Lucas, A.M. and P.R. Stettenheim, 1972. Uropygial Gland. In: Avian Anatomy: Integument, U.S. Agricultural Research Service, Washington, pp: 613-626.

Mclelland, J., 1993. Digestive System. In: Handbook of Avian Anatomy: Nomina Anatomica Avium, Baumel, J.J. (Ed.), Nuttall Ornithological Club, Cambridge, pp: 301-327.

Mitchell, M.A. and M.W. Smith, 1991. The effects of genetic selection for increased growth rate on mucosal and muscle weights in the different regions of the small intestine of the domestic fowl (Gallus domesticus). Comparative Biochem. Physiol. Part A: Physiol., 99: 251-258. DOI: 10.1016/0300-9629(91)90268-H

Montalti, D. and A. Saliban, 2000. Uropygial gland size and avian habitat. Ornitol. Neotropical, 11:297-306.

Montalti, D. Ontalti, A.M. Gutierrez and A. Salibian, 1998. Técnica quirúrgica para la ablación de la glándula uropigia en la paloma casera Columba livia. Rev. Brasileira Biol., 58: 193-196. 
Montalti, D., A.M. Gutiérrez, G.R. Reboredo and A. Salibián, 2006. Removal of the uropygial gland does not affect serum lipids, cholesterol and calcium levels in the rock pigeon Columba livia. Acta Biol. Hungarica, 57: 295-300. PMID: 17048693

Moyer, B.R., A.N. Rock and D.H. Clayton, 2003. Experimental test of the importance of preen oil in rock doves (Columba livia). Auk, 120: 490-496. DOI: $10.2307 / 4090201$

Muelling, C. and S. Buda, 2002. Morphology and Function of the Digestive System in Turkey. Proceedings of the 4th International Symposium on Turkey Diseases, (STD’02), DVG, Gießen, pp: 89-96.

Nasrin, M., M.N.H. Siddiqi, M.A. Masum and M.A. Wares, 2012. Gross and histological studies of digestive tract of broilers during postnatal growth and development. J. Bangladesh Agric. Uni., 10: 69-77. DOI: 10.3329/jbau.v10i1.120VACAC96

Nickel, R., A. Schummer and E. Seiferle, 1977. The Skin Anatomy of the Domestic Birds. 1st Edn., Verlag Paul Parey, Berlin.

Qureshi, M.A. and G.B. Havenstein, 1994. A comparison of the immune performance of a 1991 commercial broiler with a 1957 randombred strain when fed "Typical" 1957 and 1991 broiler diets. Poultry Sci., 73: 1805-1812. DOI: 10.3382/ps.0731805

Robinson, F.E., H.L. Classen, J.A. Hanson and D.K. Onderka, 1992. Growth performance, feed efficiency and the incidence of skeletal and metabolic disease in full-fed and feed-restricted broiler and roaster chickens. J. Applied Poultry Res., 1: 33-41. DOI: 10.1093/japr/1.1.33

Rosenberg, L.E., 1941. Microanatomy of the duodenum of the turkey. Hilgardia, 13: 623-654. DOI: $10.3733 /$ hilg.v13n11p623
Sawad, 2006. Discerning adaptive value of seasonal variation in preen waxes: Comparative and experimental approaches. Acta Zool. Sinica, 52: $272-275$

Shawkey, M.D., S.R. Pillai and G.E. Hill, 2003. Chemical warfare? Effects of uropygial oil on feather-degrading bacteria. J. Avian Biol., 34: 345-349. DOI: 10.1111/j.0908-8857.2003.03193.x

Soler, J.J., J.M. Peralta-Sánchez, A.M. Martin-Platero, M. Martin-Vivaldi and M. Martínez-Bueno et al., 2012. The evolution of size of the uropygial gland: Mutualistic feather mites and uropygial secretion reduce bacterial loads of eggshells and hatching failures of European birds. J. Evolutionary Biol., 25: 1779-1791. DOI: $10.1111 / \mathrm{j} .1420-9101.2012 .02561$

Steel, R.G.D. and J.H. Torrie, 1980. Principles and Procedures of Statistics: A Biometrical Approach. 2nd Edn., McGraw-Hill, Tokyo, ISBN-10: 0070665818, pp: 633.

Taylor, R.D. and G.P.D. Jones, 2004. The incorporation of whole grain into pelleted broiler chicken diets. II. Gastrointestinal and digesta characteristics. Br. Poultry. Sci., 45: 237-246. DOI: $10.1080 / 00071660410001715849$

Vincze, O., C.I. Vágási, I. Kovács, I. Galván and P.L. Pap, 2013. Sources of variation in uropygial gland size in European birds. Biol. J. Linnean Society, 110: 543-563. DOI: $10.1111 /$ bij. 12139

Wepruk, J. and S. Church, 2003. Balancing production and welfare: Complex animal care issues. Alberta Farm Animal Care (AFAC) Association. 